\title{
Increased risk of diabetes with statin treatment is associated with impaired insulin sensitivity and insulin secretion: a 6 year follow-up study of the METSIM cohort
}

\author{
Henna Cederberg • Alena Stančáková • Nagendra Yaluri • \\ Shalem Modi • Johanna Kuusisto • Markku Laakso
}

Received: 8 October 2014 / Accepted: 30 January 2015 /Published online: 10 March 2015

(C) Springer-Verlag Berlin Heidelberg 2015

\begin{abstract}
Aims/hypothesis The aim of this work was to investigate the mechanisms underlying the risk of type 2 diabetes associated with statin treatment in the population-based Metabolic Syndrome in Men (METSIM) cohort.

Methods A total of 8,749 non-diabetic participants, aged 45-73 years, were followed up for 5.9 years. New diabetes was diagnosed in 625 men by means of an OGTT, $\mathrm{HbA}_{1 \mathrm{c}}$ $\geq 6.5 \%$ (48 mmol $/ \mathrm{mol})$ or glucose-lowering medication started during the follow-up. Insulin sensitivity and secretion were evaluated with OGTT-derived indices.

Results Participants on statin treatment $(N=2,142)$ had a $46 \%$ increased risk of type 2 diabetes (adjusted HR 1.46 [95\% CI $1.22,1.74])$. The risk was dose dependent for simvastatin and atorvastatin. Statin treatment significantly increased $2 \mathrm{~h}$ glucose (2hPG) and glucose AUC of an OGTT at follow-up, with a nominally significant increase in fasting plasma glucose (FPG). Insulin sensitivity was decreased by $24 \%$ and insulin secretion by $12 \%$ in individuals on statin treatment (at FPG and $2 \mathrm{hPG}<5.0 \mathrm{mmol} / \mathrm{l}$ ) compared with individuals without statin treatment $(p<0.01)$. Decreases in insulin sensitivity and insulin secretion were dose dependent for simvastatin and atorvastatin.
\end{abstract}

Electronic supplementary material The online version of this article (doi:10.1007/s00125-015-3528-5) contains peer-reviewed but unedited supplementary material, which is available to authorised users.

H. Cederberg $\cdot$ J. Kuusisto $\cdot$ M. Laakso $(\bowtie)$

Institute of Clinical Medicine, Internal Medicine,

University of Eastern Finland and Kuopio University Hospital,

P.O. Box 1627, 70211 Kuopio, Finland

e-mail: markku.laakso@kuh.fi

A. Stančáková $\cdot$ N. Yaluri $\cdot$ S. Modi

Institute of Clinical Medicine, Internal Medicine,

University of Eastern Finland, Kuopio, Finland
Conclusions/interpretation Statin treatment increased the risk of type 2 diabetes by $46 \%$, attributable to decreases in insulin sensitivity and insulin secretion.

Keywords 2-h glucose $\cdot$ Fasting glucose $\cdot \mathrm{HbA}_{1 \mathrm{c}} \cdot$ Insulin resistance $\cdot$ Insulin sensitivity $\cdot$ Statin . Type 2 diabetes

$\begin{array}{ll}\text { Abbreviations } \\ \text { 2hPG } & 2 \text { h plasma glucose } \\ \text { CVD } & \text { Cardiovascular disease } \\ \text { DI } & \text { Disposition index } \\ \text { FPG } & \text { Fasting plasma glucose } \\ \text { HOMA-B } & \text { HOMA of beta cell function } \\ \text { ISI } & \text { Insulin sensitivity index } \\ \text { METSIM } & \text { Metabolic syndrome in men } \\ \text { WOSCOPS } & \text { West of Scotland Coronary Prevention Study }\end{array}$

Introduction

Statin treatment is effective in the primary and secondary prevention of cardiovascular disease (CVD) events in individuals with and without diabetes [1,2] and is generally safe and well tolerated [2]. In the West of Scotland Coronary Prevention Study (WOSCOPS) pravastatin treatment decreased the risk of diabetes by $30 \%$ [3]. Emerging evidence, however, suggests that treatment with other statins slightly increases the risk of type 2 diabetes [4-7]. In pooled data from 13 trials statin therapy was associated with a $9 \%$ increased risk of diabetes [8] and this effect was age and dose dependent $[8,9]$. Previous population-based studies have reported a $10-22 \%$ increased risk of diabetes with statins [10-12].

Mechanisms underlying the association of statin therapy with diabetes remain unclear [13]. Type 2 diabetes develops as a combination of insulin resistance and progressive beta 
cell dysfunction, the latter being required for the conversion to overt diabetes. Studies of the effects of statin treatment on insulin sensitivity are conflicting and are generally small in size [14-18]. Treatment with simvastatin and rosuvastatin has decreased insulin sensitivity, whereas treatment with pravastatin has improved insulin sensitivity $[19,20]$. Statininduced decrease in insulin secretion has been reported in some [21], but not all, in vitro studies [22]. Human studies on statin-induced changes in glucose-stimulated insulin secretion are lacking.

There are limitations in previous studies that have evaluated the diabetogenic impact of statin treatment. Study populations have been selective, especially in statin trials which have included participants at high risk of CVD [6-9]. Therefore, the risk of diabetes in clinical trials is likely to differ from that in the general population. Very often in previous studies the diagnosis of diabetes has been based on self-reported diabetes or fasting glucose measurement $[5,6,8,9]$, underestimating incident diabetes. There have been no previous studies wherein the diagnosis of diabetes has been based on the current diagnostic criteria for diabetes: fasting glucose, an OGTT or $\mathrm{HbA}_{1 \mathrm{c}}$. Finally, there are no population-based studies evaluating the mechanisms underlying the diabetogenic effects of statins. Therefore, we investigated the effects of statin treatment on the risk of type 2 diabetes and deterioration towards hyperglycaemia in 8,749 non-diabetic men in a 6 year follow-up of the populationbased Metabolic Syndrome in Men (METSIM) study. We also investigated the mechanisms of statin-induced diabetes by evaluating changes in insulin resistance and insulin secretion.

\section{Methods}

Participants and clinical measurement at the baseline study The METSIM study was performed in 2005-2010 at the Clinical Research Unit of the University of Kuopio and included 10,197 men, aged 45-73 years, randomly selected from the population register of Kuopio, Eastern Finland (population 95,000) [23]. An OGTT (75 g of glucose, glucose and insulin measurements at 0,30 and $120 \mathrm{~min}$ ) was performed, and glucose tolerance was classified according to the American Diabetes Association criteria [24]. Participants with previously diagnosed type 1 diabetes $(n=25)$, newly $(n=649)$ or previously diagnosed type 2 diabetes $(n=763)$ or those without an OGTT at baseline $(n=11)$ were excluded. A total of 8,749 men without diabetes at baseline were included in the statistical analyses (age $57 \pm 7$ years, BMI $26.8 \pm 3.8 \mathrm{~kg} / \mathrm{m}^{2}$, mean \pm SD).

Participants and clinical measurements at the follow-up study A follow-up started in 2010 and so far 5,419 individuals have participated. The study protocol and measurements are identical to those of the baseline study.
Diagnosis of new type 2 diabetes Out of 8,749 non-diabetic participants at baseline, 625 developed type 2 diabetes during a 5.9 year follow-up study. Diagnosis of type 2 diabetes was based on the following criteria: (1) fasting plasma glucose $(\mathrm{FPG}) \geq 7.0 \mathrm{mmol} / 1,2 \mathrm{~h}$ plasma glucose $(2 \mathrm{hPG})$ $\geq 11.1 \mathrm{mmol} / \mathrm{l}$ in an OGTT or $\mathrm{HbA}_{1 \mathrm{c}} \geq 6.5 \%(48 \mathrm{mmol} / \mathrm{mol})$ among 4,806 non-diabetic individuals who participated in the ongoing METSIM follow-up study in 2010-2014 (327 cases of new diabetes); (2) glucose-lowering medication started between the baseline study and 31 December 2013 ( $n=261$ cases of new diabetes; information obtained from the National Drug Reimbursement registry for all 8,749 non-diabetic participants); (3) type 2 diabetes diagnosed by physician as per medical records and/or FPG $\geq 7.0 \mathrm{mmol} / 1,2 \mathrm{hPG} \geq 11.1 \mathrm{mmol} / \mathrm{l}$ or $\mathrm{HbA}_{1 \mathrm{c}} \geq 6.5 \%$ (48 mmol/mol) in outpatient/primary care laboratory measurements ( $n=37$ cases of new diabetes) and the lack of symptoms and signs indicating type 1 diabetes. Of the diabetes diagnoses in the METSIM follow-up study, 22.6\% were based on FPG alone, $24.9 \%$ on $2 \mathrm{hPG}$ alone, $31.6 \%$ on $\mathrm{HbA}_{1 \mathrm{c}}$ alone and $20.8 \%$ on different combinations of these criteria. The study was approved by the Ethics Committee of the University of Eastern Finland and Kuopio University Hospital and conducted in accordance with the Helsinki Declaration. All study participants gave written informed consent.

Statin treatment A total of 2,142 (24.5\%) of the 8,749 nondiabetic men were on statin medication at baseline $(65.9 \%$ on simvastatin, $18.1 \%$ on atorvastatin, $8.6 \%$ on rosuvastatin, $3.8 \%$ on fluvastatin, $2.3 \%$ on lovastatin and $1.3 \%$ on pravastatin).

Measurements Height and weight were measured to the nearest $0.5 \mathrm{~cm}$ and $0.1 \mathrm{~kg}$, respectively. BMI was calculated as weight $(\mathrm{kg})$ divided by height $(\mathrm{m})$ squared. Waist circumference was measured at the midpoint between the lateral iliac crest and lowest rib. Smoking status was defined as current smoking (yes vs no). Family history of diabetes (yes vs no) was defined as a first-degree or second-degree relative having diabetes vs no family history of diabetes. Physical activity (physically active vs inactive) refers to leisure-time exercise (physically active, regular exercise [at least $30 \mathrm{~min}$ once or twice a week] vs physically inactive, occasional exercise or no exercise). Alcohol intake was defined as total alcohol intake in grams per week. The use of beta-blockers and diuretics at baseline was recorded (yes vs no). CVD at baseline was defined as a history of non-fatal myocardial infarction or stroke.

Laboratory measurements Plasma glucose was measured by enzymatic hexokinase photometric assay (Konelab Systems reagents; Thermo Fisher Scientific, Vantaa, Finland). $\mathrm{HbA}_{1 \mathrm{c}}$ was analysed with a Tosoh G7 glycohaemoglobin analyser (Tosoh Bioscience, San Francisco, CA, USA). Plasma insulin 
concentrations were measured by a luminometric immunoassay measurement (ADVIA Centaur Insulin IRI, no. 02230141; Siemens Medical Solutions Diagnostics, Tarrytown, NY, USA). LDL-cholesterol, HDL-cholesterol and total triacylglycerols were measured by enzymatic colorimetric tests (Konelab Systems reagents).

Calculations The trapezoidal method was used to calculate the glucose and insulin AUCs in an OGTT based on samples collected at 0,30 and $120 \mathrm{~min}$. The Matsuda index of insulin sensitivity (ISI) was calculated as $10,000 / \sqrt{ }$ (fasting insulin $\times$ fasting glucose $\times$ mean insulin during OGTT $\times$ mean glucose in an OGTT), and by HOMA-IR [25]. Disposition index (DI) was calculated as a product of insulin sensitivity and insulin secretion (Matsuda ISI $\times$ insulin $\mathrm{AUC}_{0-30 \mathrm{~min}} /$ glucose $\mathrm{AUC}_{0-30 \mathrm{~min}}$ ) and insulin secretion by HOMA of beta cell function (HOMA-B) as previously reported [23].

Statistical analysis Statistical analyses were conducted using the SPSS version 19 (SPSS, Chicago, IL, USA). BMI, waist circumference, total cholesterol, LDL-cholesterol, HDL-cholesterol, total triacylglycerols, glucose and $\mathrm{HbA}_{1 \mathrm{c}}$ levels, Matsuda ISI and DI were log-transformed to correct for their skewed distribution. Baseline characteristics of the groups were compared using $t$ test or $\chi^{2}$ test. The $p$ value for per cent differences between statin and no statin groups was calculated using the general linear or logistic regression models, as appropriate (Table 1). HRs for the risk of diabetes were calculated with Cox regression (Table 2, electronic supplementary material [ESM] Table 1). Risk of diabetes according to the type of statin (simvastatin, atorvastatin, or other statins [including rosuvastatin, pravastatin, fluvastatin and lovastatin] vs no statin), the dose of statin, and changes in statin treatment during the study were calculated with Cox regression (Fig. 1, ESM Fig. 1). The association of statin treatment with FPG, $2 \mathrm{hPG}$ and glucose AUC at follow-up was evaluated with linear regression analysis $(N=4,679$ non-diabetic participants at baseline had follow-up data available, excluding individuals diagnosed with diabetes between baseline and follow-up). Adjustments were made in models 2-14 (Table 2) for age, BMI, waist circumference, current smoking, physical activity, alcohol intake, family history of diabetes, use of beta-blockers and use of diuretics, as well as for the length of follow-up time (in months) in linear regression analysis. Additional adjustments were made for FPG, 2hPG, Matsuda ISI, DI, LDLcholesterol, HDL-cholesterol, total triacylglycerols and CVD and the changes in LDL-cholesterol, HDL-cholesterol, total triacylglycerols and BMI. In Table 2, $p<0.004$ was considered as statistically significant given the 12 different models tested (Bonferroni correction for multiple testing) and $p<0.05$ was considered nominally significant. Differences in Matsuda ISI and DI (Table 3) and HOMA-IR (ESM Table 2) in non-diabetic individuals at baseline treated with simvastatin or atorvastatin vs no statin and in individuals receiving low-dose or high-dose atorvastatin or simvastatin vs no statin were compared with the ANOVA post hoc tests. Matsuda ISI and DI between the individuals with and without statin therapy in categories of FPG and 2hPG were compared using the $t$ test (unadjusted model, Fig. 2), and linear regression (adjusted for age, BMI, waist circumference, current smoking, physical activity, alcohol intake and family history of diabetes) (ESM Table 3), and similarly for HOMA-IR and HOMA-B (ESM Table 4).

\section{Results}

Risk of type 2 diabetes with statin treatment At entry individuals who developed diabetes were older, more obese, less physically active, had lower levels of HDL-cholesterol and had higher levels of total triacylglycerols, FPG, 2hPG and $\mathrm{HbA}_{1 \mathrm{c}}$. Additionally, they were more insulin resistant and had lower insulin secretion than individuals who did not develop diabetes, independently of statin treatment (Table 1).

Participants treated with statins developed diabetes more often than participants without statin treatment $(11.2 \%$ vs $5.8 \%, p<0.001)$. Statin treatment increased the risk of type 2 diabetes by twofold during the follow-up (HR 2.01 [95\% CI 1.71, 2.36]) (Table 2). After adjustment for age, BMI, waist circumference, physical activity, smoking, alcohol intake, family history of diabetes and beta-blocker and diuretic treatment, the risk was $1.46(1.22,1.74)$. Adjustment for FPG, 2hPG, Matsuda ISI, DI, LDL-cholesterol, HDL-cholesterol, total triacylglycerols and CVD at baseline and changes in LDL-cholesterol and HDL cholesterol, total triacylglycerols and BMI during the follow-up slightly attenuated, but did not abolish, the association of statin treatment with newonset diabetes. Adjustment for glucose tolerance status at baseline had a similar effect as adjustment for FPG or $2 \mathrm{hPG}$ at baseline (not shown).

Effects of different statins and statin doses on the risk of diabetes Both simvastatin and atorvastatin increased the risk of type 2 diabetes compared with no statin treatment (HR 2.11 [95\% CI 1.76, 2.54] and HR 1.50 [95\% CI 1.30, 1.73], respectively), and these associations remained significant after adjustment for confounding factors (HR 1.49 [95\% CI 1.22, 1.83], and HR 1.21 [95\% CI 1.04, 1.40], respectively). Other statins did not increase the risk of diabetes (Fig. 1b). The risk of diabetes was dose dependent for both simvastatin and atorvastatin (Fig. 1c, d). After the adjustment for confounding factors, both simvastatin (high and low dose) and atorvastatin (high dose) significantly increased the risk of diabetes (simvastatin HR 1.44 [95\% CI 1.23, 1.68] and 1.28 [95\% CI 1.01, 1.62] for high and low dose, respectively, and atorvastatin HR 1.37 [95\% CI 1.14, 1.65]). 


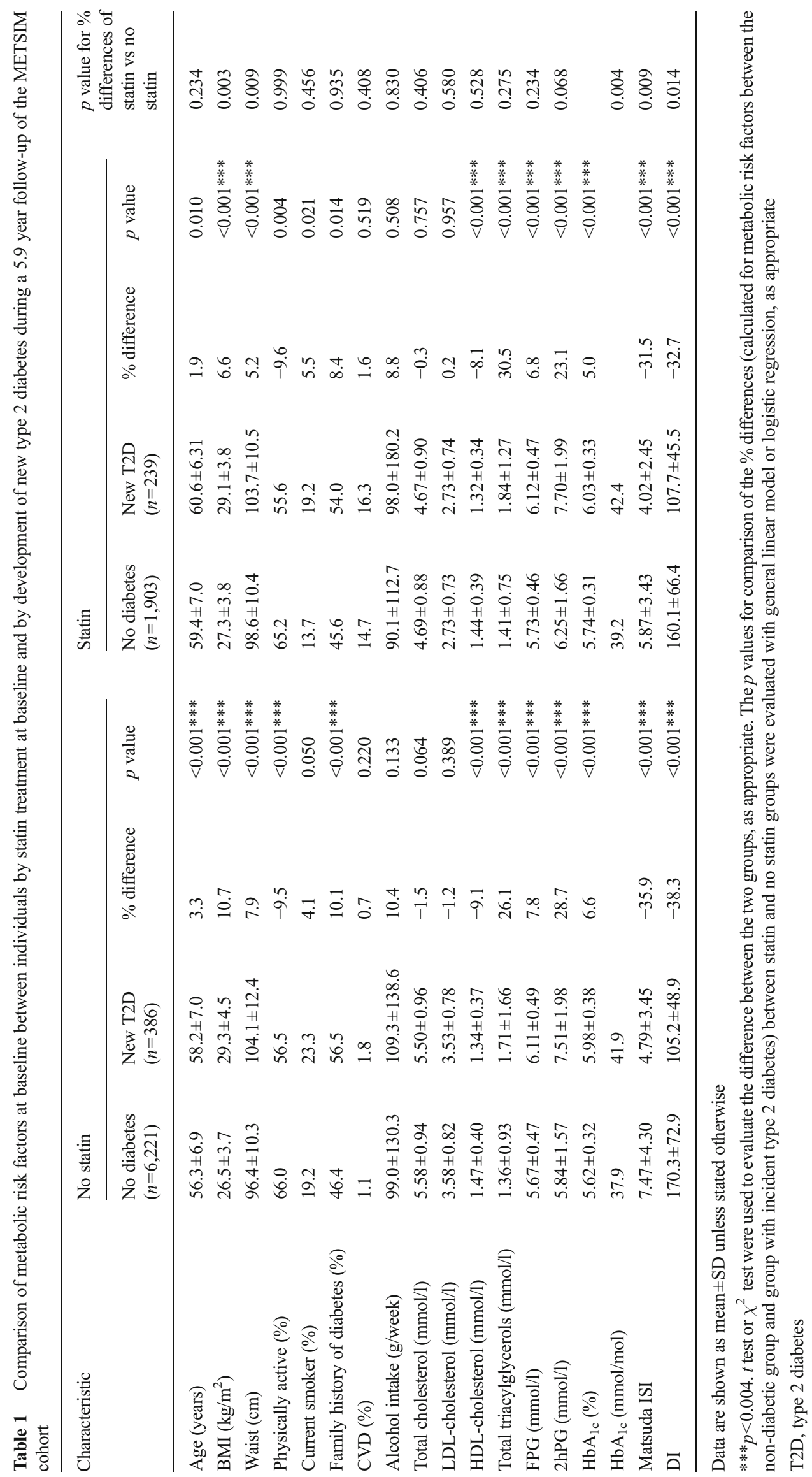


Fig. 1 Risk of type 2 diabetes by statin treatment during the 5.9 year follow-up. (a) Total cohort (625 cases of new type 2 diabetes and 8,124 non-diabetic controls). Black line, statin treatment at baseline $(n=2,141)$; grey line, no statin treatment at baseline $(n=6,607)$. (b) Risk by different statins. Black continuous line, atorvastatin $(n=388)$; black dotted line, simvastatin $(n=$ $1,409)$; grey dotted line, other statins (including rosuvastatin, pravastatin, fluvastatin and lovastatin, $n=342$ ); grey continuous line, no statin treatment. (c) Risk by dose of simvastatin. Black line, high dose (40 or $80 \mathrm{mg} /$ day, $n=385$ ); dotted line, low dose (10 or $20 \mathrm{mg} /$ day, $n=971$ ); grey line, no statin treatment. (d) Risk by dose of atorvastatin. Black line, high dose (20 or $40 \mathrm{mg} /$ day, $n=197$ ); dotted line, low dose $(10 \mathrm{mg} / \mathrm{day}$, $n=175)$; grey line, no statin treatment. Unadjusted Cox regression analysis
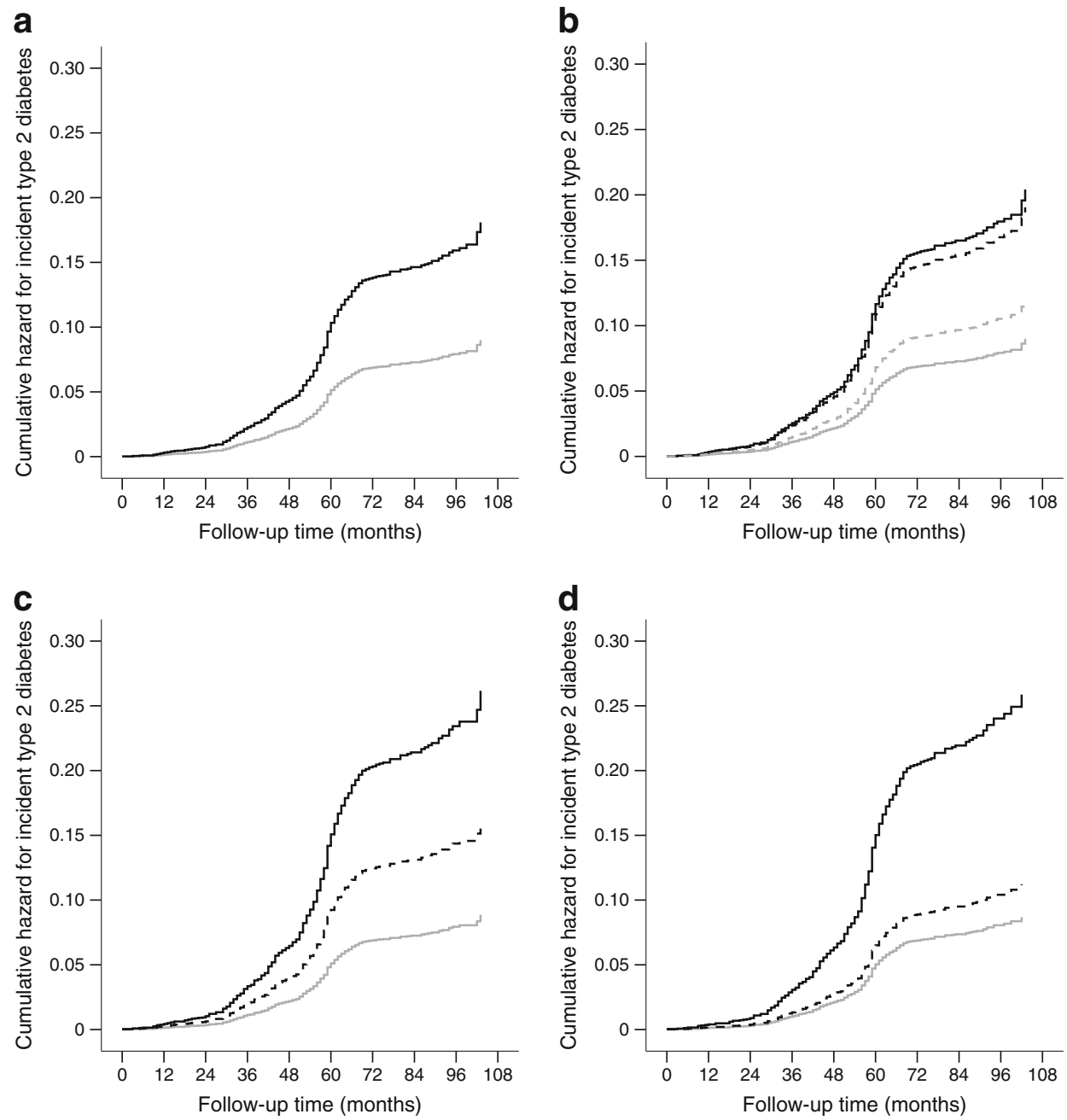

Time-dependency of the effect of statin treatment on the risk of incident diabetes The effects of changes in statin medication were evaluated in 4,786 participants with information on statin treatment at both baseline and follow-up. Subgroup analysis of participants who continued statin treatment from baseline to follow-up, initiated statin medication after baseline or discontinued statin medication before the follow-up study showed some evidence of time dependency of the risk (ESM Fig. 1 and ESM Table 1). The association of statin treatment with incident diabetes, when statin was discontinued after baseline, was attenuated after adjustment for confounding factors (ESM Table 1, adjusted model).

Worsening of hyperglycaemia with statin treatment Statin treatment significantly increased the levels of $2 \mathrm{hPG}$ and the glucose AUC at follow-up ( $p=0.001$ and $p<0.001$, respectively), and nominally the levels of FPG at follow-up ( $p=$ 0.037 ) after adjustment for confounding factors (Table 2). The association of statin treatment with $2 \mathrm{hPG}$ at follow-up was stronger than that with FPG; the association remained nominally significant after the adjustment for FPG at baseline,
Matsuda ISI and DI (Models 3-6) but was abolished after the adjustment for $2 \mathrm{hPG}$ at baseline. Adjustment for CVD, LDLcholesterol, HDL-cholesterol and total triacylglycerols and change in BMI did not attenuate the associations of statin treatment with glycaemia (Models 7-14).

Association of statin treatment with insulin secretion and insulin sensitivity Statin treatment was associated with a $24.3 \%$ reduced insulin sensitivity (Matsuda ISI) in the lowest category of FPG $(<5.0 \mathrm{mmol} / \mathrm{l})$ and with a $19.5 \%$ reduced insulin sensitivity in the lowest category of $2 \mathrm{hPG}$ $(<5.0 \mathrm{mmol} / \mathrm{l})$ compared with individuals without statin treatment $(p<0.001)$ (Fig. 2 and ESM Table 3). Statin treatment reduced insulin secretion (DI) by $12.0 \%$ in the lowest category of FPG compared with individuals without statin treatment $(p<0.01)$. The reduction in Matsuda ISI in different glucose tolerance categories (from $<5.0$ to $6.9 \mathrm{mmol} / 1$ for $\mathrm{FPG}$, and from $<5.0$ to 7.99 for $2 \mathrm{hPG}$ ) remained statistically significant after adjustment for confounding factors whereas the reduction in DI in the FPG and 2hPG categories lost its statistical significance after the adjustment for confounding factors in all 
Table 2 Association of statin treatment at baseline with the risk of new diabetes and worsening of hyperglycaemia during a 5.9 year follow-up of the METSIM cohort

\begin{tabular}{|c|c|c|c|c|c|c|c|c|c|c|c|c|c|c|c|}
\hline \multirow[t]{2}{*}{ Model } & \multicolumn{3}{|c|}{ New T2D } & \multicolumn{4}{|c|}{ FPG at follow-up } & \multicolumn{4}{|c|}{$2 \mathrm{hPG}$ at follow-up } & \multicolumn{4}{|c|}{ Glucose AUC at follow-up } \\
\hline & HR & $95 \% \mathrm{CI}$ & $p$ value & $\beta$ & $B$ & SE & $p$ value & $\beta$ & $B$ & SE & $p$ value & $\beta$ & $B$ & SE & $p$ value \\
\hline 1 & 2.01 & $1.71,2.36$ & $<0.001 * * *$ & 0.056 & 0.073 & 0.019 & $<0.001 * * *$ & 0.114 & 0.528 & 0.068 & $<0.001 * * *$ & 0.108 & 38.14 & 5.13 & $<0.001 * * *$ \\
\hline 2 & 1.46 & $1.22,1.74$ & $<0.001 * * *$ & 0.024 & 0.032 & 0.020 & 0.037 & 0.044 & 0.205 & 0.071 & $0.001 * * *$ & 0.049 & 17.17 & 5.37 & $<0.001 * * *$ \\
\hline 3 & 1.35 & $1.13,1.62$ & $0.001 * * *$ & -0.001 & -0.002 & 0.018 & 0.745 & 0.037 & 0.173 & 0.071 & 0.006 & 0.032 & 11.42 & 5.18 & 0.007 \\
\hline 4 & 1.38 & $1.16,1.64$ & $<0.001 * * *$ & 0.017 & 0.023 & 0.020 & 0.101 & 0.021 & 0.098 & 0.062 & 0.058 & 0.031 & 10.82 & 4.96 & 0.006 \\
\hline 5 & 1.35 & $1.13,1.61$ & $0.001 * * *$ & 0.008 & 0.011 & 0.020 & 0.332 & 0.025 & 0.117 & 0.070 & 0.044 & 0.026 & 9.17 & 5.24 & 0.025 \\
\hline 6 & 1.44 & $1.20,1.71$ & $<0.001 * * *$ & 0.009 & 0.012 & 0.019 & 0.274 & 0.027 & 0.124 & 0.066 & 0.023 & 0.028 & 9.89 & 4.81 & 0.009 \\
\hline 7 & 1.40 & $1.16,1.70$ & $0.001 * * *$ & 0.031 & 0.041 & 0.022 & 0.015 & 0.054 & 0.251 & 0.077 & $<0.001 * * *$ & 0.062 & 21.65 & 5.79 & $<0.001 * * *$ \\
\hline 8 & 1.37 & $1.11,1.70$ & $0.003 * * *$ & 0.023 & 0.031 & 0.020 & 0.045 & 0.048 & 0.223 & 0.072 & $0.001 * * *$ & 0.050 & 17.65 & 5.43 & $<0.001 * * *$ \\
\hline 9 & 1.46 & $1.22,1.74$ & $<0.001 * * *$ & 0.026 & 0.034 & 0.020 & 0.027 & 0.047 & 0.218 & 0.071 & $0.001 * * *$ & 0.051 & 17.79 & 5.37 & $<0.001 * * *$ \\
\hline 10 & 1.30 & $1.06,1.60$ & 0.013 & 0.025 & 0.032 & 0.020 & 0.035 & 0.044 & 0.203 & 0.071 & $0.001 * * *$ & 0.049 & 17.16 & 5.37 & $<0.001^{* * *}$ \\
\hline 11 & 1.47 & $1.23,1.76$ & $<0.001 * * *$ & 0.022 & 0.029 & 0.020 & 0.053 & 0.040 & 0.187 & 0.070 & $0.003 * * *$ & 0.045 & 15.70 & 5.32 & $<0.001 * * *$ \\
\hline 12 & 1.30 & $1.06,1.60$ & 0.013 & 0.022 & 0.029 & 0.020 & 0.048 & 0.042 & 0.195 & 0.071 & $0.002 * * *$ & 0.047 & 16.38 & 5.35 & $<0.001 * * *$ \\
\hline 13 & 1.47 & $1.23,1.76$ & $<0.001 * * *$ & 0.033 & 0.044 & 0.020 & 0.013 & 0.049 & 0.229 & 0.072 & $0.001 * * *$ & 0.054 & 19.105 & 5.462 & $<0.001 * * *$ \\
\hline 14 & 1.28 & $1.04,1.58$ & $<0.001 * * *$ & 0.020 & 0.027 & 0.020 & 0.066 & 0.040 & 0.185 & 0.070 & $0.003 * * *$ & 0.044 & 15.631 & 5.307 & $<0.001 * * *$ \\
\hline
\end{tabular}

Cox regression analysis was applied to evaluate the risk of diabetes ( 625 cases vs 8,124 non-diabetic controls). Linear regression was applied to evaluate the worsening of hyperglycaemia (4,679 non-diabetic participants at baseline; follow-up 4.3 years)

Model 1: unadjusted

Model 2: adjusted for age, BMI, waist, physical activity, smoking, alcohol, and family history of diabetes, use of beta-blockers, use of diuretics

Model 3: Model $2+$ adjusted for FPG at baseline

Model 4: Model $2+$ adjusted for $2 \mathrm{hPG}$ at baseline

Model 5: Model $2+$ adjusted for Matsuda ISI at baseline

Model 6: Model $2+$ adjusted for DI at baseline

Model 7: Model $2+$ adjusted for LDL cholesterol level at baseline

Model 8: Model $2+$ adjusted for change in LDL-cholesterol level between baseline and follow-up

Model 9: Model $2+$ adjusted for HDL-cholesterol level at baseline

Model 10: Model $2+$ adjusted for change in HDL-cholesterol level between baseline and follow-up

Model 11: Model $2+$ adjusted for total triacylglycerol level at baseline

Model 12: Model $2+$ adjusted for change in total triacylglycerol level between baseline and follow-up

Model 13: Model $2+$ adjusted for CVD at baseline

Model 14: Model 2 + adjusted for change in BMI between baseline and follow-up

Linear regression also adjusted for follow-up time (in months) in Models 2-14

$* * * p<0.004$

T2D, type 2 diabetes

categories except for FPG $<5.0 \mathrm{mmol} / \mathrm{l}$ (ESM Table 3). Similar reductions in insulin sensitivity across the glucose categories as for Matsuda ISI were observed for HOMA-IR (ESM Table 4). A decrease in HOMA-B across the fasting glucose categories was parallel to an increase in HOMA-IR, but across the $2 \mathrm{hPG}$ categories there was a small compensatory increase in HOMA-B (ESM Table 4).

The effect of different statins and statin doses on insulin sensitivity and insulin secretion Treatment with either simvastatin or atorvastatin was associated with significant reduction in Matsuda ISI (21.9 and 24.4\%, respectively) and DI ( 7.6 and $7.4 \%$, respectively) compared with no statin treatment (Table 3). There was a significant decrease in insulin sensitivity with an increasing dose of simvastatin (low dose, 20.8\%; high dose, 25.4\%) and atorvastatin $(16.6 \%$ and $30.2 \%$, respectively) (Table 3). Similar reductions in insulin sensitivity were observed for HOMA-IR (ESM Table 2). Corresponding decreases in insulin secretion were considerably smaller for both simvastatin (low dose, 6.6\%; high dose, 9.8\%) and atorvastatin (3.4\% and $10.5 \%$, respectively). 
Table 3 The association of simvastatin and atorvastatin treatment at baseline and their doses with insulin sensitivity (Matsuda ISI) and insulin secretion (DI) in non-diabetic participants in the cross-sectional METSIM study

\begin{tabular}{|c|c|c|c|c|c|c|c|c|c|c|}
\hline \multirow[t]{2}{*}{ Treatment/dose } & \multicolumn{5}{|c|}{ Matsuda ISI } & \multicolumn{5}{|l|}{ DI } \\
\hline & $n$ & Mean & SD & $\%$ change & $p$ value (vs no statin) & $n$ & Mean & SD & $\%$ change & $p$ value (vs no statin) \\
\hline No statin & 6,569 & 7.31 & 4.3 & & - & 6,569 & 166.5 & 73.3 & & - \\
\hline Simvastatin & 1,397 & 5.71 & 3.48 & -21.9 & $<0.001 * * *$ & 1,397 & 153.8 & 66.4 & -7.6 & $<0.001 * * *$ \\
\hline Atorvastatin & 388 & 5.53 & 3.21 & -24.4 & $<0.001 * * *$ & 388 & 154.1 & 71 & -7.4 & $<0.001 * * *$ \\
\hline \multicolumn{11}{|l|}{ Simvastatin dose (mg/day) } \\
\hline Low dose $(10$ or 20$)$ & 960 & 5.79 & 3.49 & -20.8 & $<0.001 * * *$ & 960 & 155.5 & 66.8 & -6.6 & $<0.001 * * *$ \\
\hline High dose (40 or 80 ) & 384 & 5.45 & 3.35 & -25.4 & $<0.001 * * *$ & 384 & 150.1 & 67.0 & -9.8 & $<0.001^{* * *}$ \\
\hline \multicolumn{11}{|c|}{ Atorvastatin dose (mg/day) } \\
\hline Low dose (10) & 175 & 6.10 & 3.46 & -16.6 & $0.001 * * *$ & 175 & 160.9 & 72.4 & -3.4 & 0.580 \\
\hline High dose (20 or 40$)$ & 197 & 5.10 & 2.95 & -30.2 & $<0.001 * * *$ & 197 & 149.1 & 71.5 & -10.5 & $<0.001^{* * *}$ \\
\hline
\end{tabular}

The reference group in each analysis is the group without statin treatment at baseline

$* * * p<0.004$

\section{Discussion}

We investigated the association of statin treatment with the risk of type 2 diabetes and the worsening of hyperglycaemia in a population-based prospective METSIM study including 8,479 non-diabetic Finnish men. Our study reports several novel findings: (1) statin therapy was associated with a $46 \%$ increased risk of type 2 diabetes after adjustment for confounding factors, suggesting a higher risk of diabetes in the general population than previously reported; (2) statin therapy was associated with a worsening of hyperglycaemia, especially $2 \mathrm{~h}$ glucose; (3) statin therapy was associated with a $24 \%$ reduction in insulin sensitivity and $12 \%$ reduction in insulin secretion compared with individuals without statin therapy
Fig. 2 Matsuda ISI and DI across the categories of FPG $(\mathbf{a}, \mathbf{b})$ and $2 \mathrm{hPG}(\mathbf{c}, \mathbf{d})$ in the METSIM study participants with (black bars, $n=2,142)$ and without statin treatment (white bars, $n=6,607$ ) at baseline. Data are unadjusted means (SEM). $* p<0.05$, $* * p<0.01$ and $* * * p<0.001$, for statin vs no statin
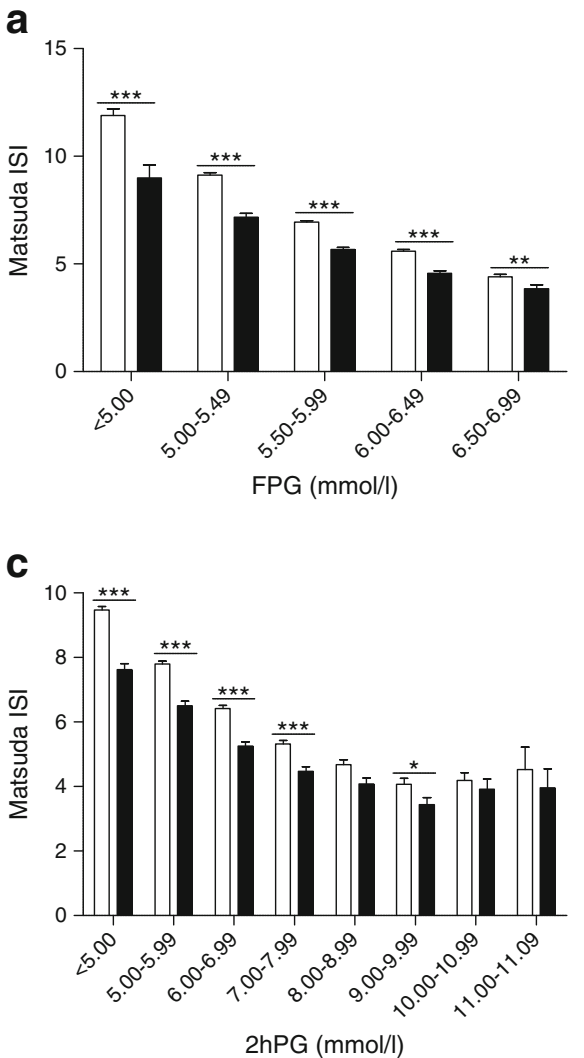
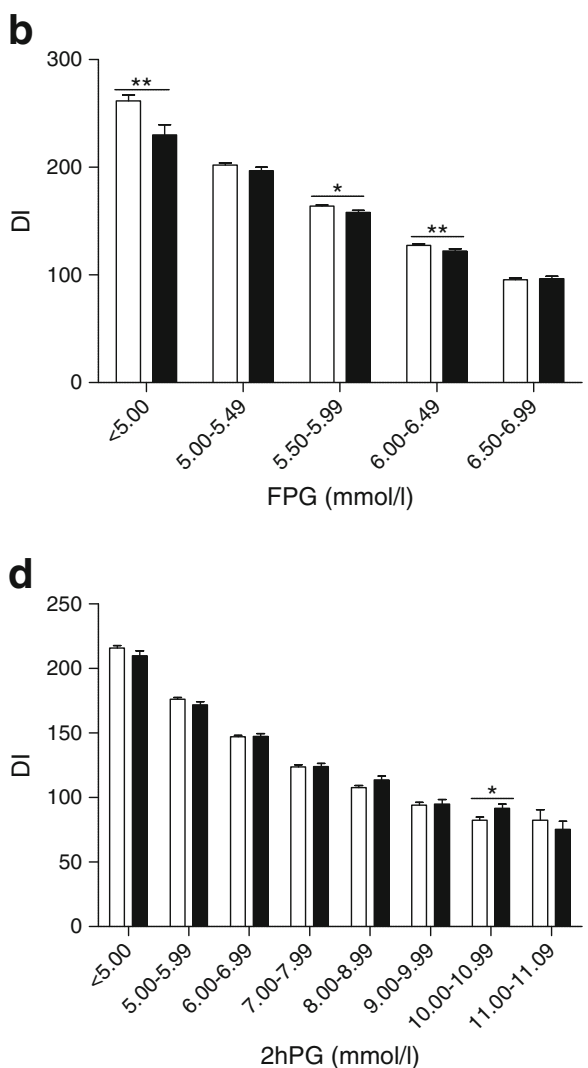
and (4) both simvastatin and atorvastatin treatment was associated with reduced insulin sensitivity and insulin secretion in a dose-dependent manner.

Statins and the risk of type 2 diabetes and hyperglycaemia In our study statin therapy was associated with a higher risk of diabetes $(46 \%)$ than previously reported. Based on registry or prescription-based data, an Irish study reported a $20 \%$ increase, a Canadian study a $10-22 \%$ increase and a Taiwanese study a $15 \%$ increase in the risk of type 2 diabetes associated with statin therapy [10-12]. In the Women's Health Initiative study statin therapy was associated with a $48 \%$ increase in the risk of self-reported diabetes [5]. In a collaborative meta-analysis of 13 randomised statin trials $(N=91,140$ participants), statin therapy was associated with a $9 \%$ increased risk for type 2 diabetes, based on FPG or physicianreported diagnosis of diabetes [8]. The higher risk of type 2 diabetes found in our study suggests that previous studies may have underestimated the significance of statin-induced diabetes. In our study $56.5 \%$ of the diabetes diagnoses were made according to $2 \mathrm{hPG}$ and/or $\mathrm{HbA}_{1 \mathrm{c}}$ criteria and therefore the use of either FPG or physician-reported diagnosis of diabetes as a sole criterion for diabetes diagnosis may significantly underestimate incident diabetes. Individuals who developed diabetes on statin therapy in our study had a similar metabolic risk factor profile at baseline to those who developed diabetes without statin therapy, suggesting that statin treatment increased the risk of diabetes independently of the risk profile of the background population. Our study is also the first to show that statin therapy was associated with the worsening of $2 \mathrm{~h}$ hyperglycaemia at follow-up. Increased levels of FPG in non-diabetic individuals receiving statin therapy have been previously reported in some [26, 27], but not all [20], studies; however, no previous study has reported significant changes in $2 \mathrm{hPG}$ level in people receiving statin treatment.

Differences in the risk of diabetes with varying statins and statin doses In our study atorvastatin and simvastatin were the most diabetogenic and pravastatin, fluvastatin and lovastatin were less diabetogenic, in agreement with the findings reported in previous studies [28]. However, the number of participants receiving pravastatin, fluvastatin and lovastatin was too small to reliably estimate their individual effects on the risk of diabetes. The risk of type 2 diabetes in our study was increased in a dose-dependent manner by simvastatin and atorvastatin treatment in agreement with a meta-analysis of five statin trials [9]. No definite conclusions can be drawn as to the dose-dependent effect of the other statins due to a low number of participants in these subgroups.

Statin-induced diabetes: possible mechanisms The mechanisms underlying statin-induced diabetes are poorly known, but defects in insulin secretion and insulin resistance have been suggested [13, 19-21]. Our study demonstrated for the first time that one of the two mechanisms leading to incident diabetes in people receiving statin treatment was an increase in insulin resistance, reflected by elevated levels of $2 \mathrm{hPG}$. The most pronounced reduction in Matsuda ISI in people receiving statin therapy was observed at the lowest levels of glycaemia ( $\mathrm{FPG}<5.5 \mathrm{mmol} / 1$ and $2 \mathrm{hPG}<7.0 \mathrm{mmol} / \mathrm{l}$ ), indicating that the harmful effects of statin treatment are observed especially in the low normoglycaemic range. At higher glucose concentrations the difference in insulin sensitivity between individuals receiving statin treatment and those not on statins was considerably smaller probably due to glucotoxic effects of hyperglycaemia. Both simvastatin and atorvastatin were associated with a significant dose-dependent reduction in Matsuda ISI (22 and 24\%, respectively) compared with individuals who were not on statin treatment. These results are in agreement with a meta-analysis of 16 statin trials showing that simvastatin increased insulin resistance [19].

Statin treatment reduced insulin sensitivity-corrected insulin secretion Similarly to the reduction in insulin sensitivity, the reduction in insulin sensitivity-corrected insulin secretion (DI) was also greatest in the lowest category of FPG and $2 \mathrm{hPG}$ (FPG $<5.0 \mathrm{mmol} / 1$ and $2 \mathrm{hPG}<7.0 \mathrm{mmol} / \mathrm{l}$ ). The magnitude of reduction in Matsuda ISI with simvastatin and atorvastatin was approximately threefold greater than the reduction in DI, suggesting that impaired ability of beta cells to respond adequately to decreased insulin sensitivity is probably the mechanism underlying the hyperglycaemic and diabetogenic effect of simvastatin and atorvastatin.

Strengths and limitations The METSIM study is a large population-based study with detailed phenotyping for measures of glucose metabolism. Our 6 year follow-up study identified 625 new cases of type 2 diabetes among 8,749 non-diabetic participants at risk, making reliable conclusions possible. Our study included white men and therefore the applicability of these results to women or to other ethnic groups remains unknown. Insulin sensitivity and secretion were evaluated using validated surrogate indices, which are not as accurate measurements as the euglycaemic clamp or intravenous glucose tolerance test. However, these measurements are not possible to perform in large population-based studies including thousands of participants. Although our cohort was large, the power of our study to demonstrate significant associations of less frequently used statins with the risk of type 2 diabetes and underlying mechanisms was limited.

Conclusions In conclusion, our population-based METSIM study including 8,749 non-diabetic individuals at baseline showed that statin therapy was associated with a $46 \%$ increase in the risk of incident type 2 diabetes after adjustment for confounding factors. 
Funding This work has been supported by the Academy of Finland, the Finnish Diabetes Research Foundation, the Finnish Cardiovascular Research Foundation, the Strategic Research Funding from the University of Eastern Finland, Kuopio, Finland and EVO grant 5263 from the Kuopio University Hospital. The funding sources did not have any role in the study design, the collection, analysis and interpretation of data, the writing of the report or in the decision to submit the paper for publication.

Duality of interest The authors declare that there is no duality of interest associated with this manuscript.

Contribution statement $\mathrm{HC}$ researched data, contributed to the discussion and wrote the manuscript. AS, NY and SM researched data, contributed to the discussion and reviewed the manuscript. JK designed the study and reviewed the manuscript. ML designed the study, wrote the manuscript and, as a corresponding author, had full access to all the data in the study and final responsibility for the decision to submit for publication. All authors approved the final version of the manuscript.

\section{References}

1. Cholesterol Treatment Trialists (CTT) Collaborators, Kearney PM, Blackwell L et al (2008) Efficacy of cholesterol-lowering therapy in 18,686 people with diabetes in 14 randomised trials of statins: a metaanalysis. Lancet 371:117-125

2. Cholesterol Treatment Trialists (CTT) Collaborators, Baigent C, Blackwell L et al (2010) Efficacy and safety of more intensive lowering of LDL cholesterol: a meta-analysis of data from 170,000 participants in 26 randomised trials. Lancet 376:1670-1681

3. Freeman DJ, Norrie J, Sattar N et al (2001) Pravastatin and the development of diabetes mellitus: evidence for a protective treatment effect in the West of Scotland Coronary Prevention Study. Circulation 103:357-362

4. Ridker PM, Danielson E, Fonseca FA et al (2008) Rosuvastatin to prevent vascular events in men and women with elevated C-reactive protein. N Engl J Med 359:2195-2207

5. Culver AL, Ockene IS, Balasubramanian R et al (2012) Statin use and risk of diabetes mellitus in postmenopausal women in the Women's Health Initiative. Arch Intern Med 172:144-152

6. Rajpathak SN, Kumbhani DJ, Crandall J, Barzilai N, Alderman M, Ridker PM (2009) Statin therapy and risk of developing type 2 diabetes: a meta-analysis. Diabetes Care 32:1924-1929

7. Waters DD, Ho JE, DeMicco DA et al (2011) Predictors of new-onset diabetes in patients treated with atorvastatin: results from 3 large randomized clinical trials. J Am Coll Cardiol 57:1535-1545

8. Sattar N, Preiss D, Murray HM et al (2010) Statins and risk of incident diabetes: a collaborative meta-analysis of randomised statin trials. Lancet 375:735-742

9. Preiss D, Seshasai SR, Welsh P et al (2011) Risk of incident diabetes with intensive-dose compared with moderate-dose statin therapy: a meta-analysis. JAMA 305:2556-2564

10. Carter AA, Gomes T, Camacho X, Juurlink DN, Shah BR, Mamdani MM (2013) Risk of incident diabetes among patients treated with statins: population based study. BMJ 346:f2610
11. Wang KL, Liu CJ, Chao TF et al (2012) Statins, risk of diabetes, and implications on outcomes in the general population. J Am Coll Cardiol 60:1231-1238

12. Zaharan NL, Williams D, Bennett K (2013) Statins and risk of treated incident diabetes in a primary care population. $\mathrm{Br} \mathrm{J}$ Clin Pharmacol 75:1118-1124

13. Sattar N, Taskinen MR (2012) Statins are diabetogenic-myth or reality? Atheroscler Suppl 13:1-10

14. Altunbas H, Balci MK, Karayalcin U (2003) No effect of simvastatin treatment on insulin sensitivity in patients with primary hypercholesterolemia. Endocr Res 29:265-275

15. Bellia A, Rizza S, Galli A, Fabiano R, Donadel G, Lombardo MF (2010) Early vascular and metabolic effects of rosuvastatin compared with simvastatin in patients with type 2 diabetes. Atherosclerosis 210: 199-201

16. Paniagua JA, Lopez-Miranda J, Escribano A et al (2002) Cerivastatin improves insulin sensitivity and insulin secretion in early-state obese type 2 diabetes. Diabetes 51:2596-2603

17. Szendroedi J, Anderwald C, Krssak M et al (2009) Effects of highdose simvastatin therapy on glucose metabolism and ectopic lipid deposition in nonobese type 2 diabetic patients. Diabetes Care 32: 209-214

18. ter Avest E, Abbink EJ, de Graaf J, Tack CJ, Stalenhoef AF (2005) Effect of rosuvastatin on insulin sensitivity in patients with familial combined hyperlipidaemia. Eur J Clin Investig 35:558-564

19. Baker WL, Talati R, White CM, Coleman CI (2010) Differing effect of statins on insulin sensitivity in non-diabetics: a systematic review and meta-analysis. Diabetes Res Clin Pract 87:98-107

20. Moutzouri E, Liberopoulos E, Mikhailidis DP et al (2011) Comparison of the effects of simvastatin vs. rosuvastatin vs. simvastatin/ezetimibe on parameters of insulin resistance. Int J Clin Pract 65:1141-1148

21. Yada T, Nakata M, Shiraishi T, Kakei M (1999) Inhibition by simvastatin, but not pravastatin, of glucose-induced cytosolic $\mathrm{Ca}^{2+}$ signalling and insulin secretion due to blockade of L-type $\mathrm{Ca}^{2+}$ channels in rat islet $\beta$-cells. Br J Pharmacol 126:1205-1213

22. Ishikawa $M$, Okajima $F$, Inoue $N$ et al (2006) Distinct effects of pravastatin, atorvastatin, and simvastatin on insulin secretion from a beta-cell line, MIN6 cells. J Atheroscler Thromb 13:329-335

23. Stancakova A, Javorsky M, Kuulasmaa T, Haffner SM, Kuusisto J, Laakso M (2009) Changes in insulin sensitivity and insulin release in relation to glycemia and glucose tolerance in 6,414 Finnish men. Diabetes 58:1212-1221

24. American Diabetes Association (2006) Diagnosis and classification of diabetes mellitus. Diabetes Care 29(Suppl 1):S43-S48

25. Matsuda M, DeFronzo RA (1999) Insulin sensitivity indices obtained from oral glucose tolerance testing: comparison with the euglycemic insulin clamp. Diabetes Care 22:1462-1470

26. Sukhija R, Prayaga S, Marashdeh M et al (2009) Effect of statins on fasting plasma glucose in diabetic and nondiabetic patients. J Investig Med 57:495-499

27. Rautio N, Jokelainen J, Oksa H et al (2012) Do statins interfere with lifestyle intervention in the prevention of diabetes in primary healthcare? One-year follow-up of the FIN-D2D project. BMJ Open 2:e001472

28. Navarese EP, Buffon A, Andreotti F et al (2013) Meta-analysis of impact of different types and doses of statins on new-onset diabetes mellitus. Am J Cardiol 111:1123-1130 